\title{
Bendamustine combined with rituximab for patients with relapsed or refractory diffuse large $B$ cell lymphoma
}

\author{
Jeffrey L. Vacirca • Peter. I. Acs • Imad A. Tabbara • \\ Peter. J. Rosen • Peter Lee • Eric Lynam
}

Received: 11 February 2013 / Accepted: 5 August 2013 / Published online: 17 August 2013

(C) The Author(s) 2013. This article is published with open access at Springerlink.com

\begin{abstract}
Patients with relapsed or refractory diffuse large B cell lymphoma (DLBCL) are treated with salvage regimens and may be considered for high-dose chemotherapy and autologous stem cell transplantation if disease is chemosensitive. Bendamustine is active in indolent $\mathrm{B}$ cell lymphomas and chronic lymphocytic leukemia but has not been extensively studied in aggressive lymphomas. This trial examines the combination of bendamustine and rituximab in patients with relapsed and refractory DLBCL. Patients received bendamustine at $90 \mathrm{mg} / \mathrm{m}^{2}(n=2)$ or $120 \mathrm{mg} / \mathrm{m}^{2}(n=57)$ on days 1 and 2 and rituximab at $375 \mathrm{mg} / \mathrm{m}^{2}$ on day 1 every 28 days for up to 6 cycles. The study evaluated objective response rate (ORR), duration of response (DOR), progression-free survival (PFS), and treatment safety. Fifty-nine patients were treated, and 48 were evaluable for response. Median age was 74; $89 \%$ had stage III or IV disease, and $63 \%$ had high revised International Prognostic Index scores; the median number of prior therapies was 1 . Based on analysis using the intent-to-treat population, the
\end{abstract}

\author{
J. L. Vacirca $(\bowtie)$ \\ North Shore Hematology and Oncology Associates, 235 N Belle \\ Mead Road, East Setauket, NY 11733-3456, USA \\ e-mail: Jvacirca@yahoo.com \\ P. I. Acs \\ Florida Cancer Specialists, Gainesville, FL, USA \\ I. A. Tabbara \\ The George Washington University Medical Center, Washington, \\ DC, USA \\ P. J. Rosen \\ Providence Saint Joseph Medical Center, Burbank, CA, USA \\ P. Lee \\ Tower Cancer Research Foundation, Beverly Hills, CA, USA \\ E. Lynam \\ Pharmatech, Inc., Denver, CO, USA
}

ORR was $45.8 \%$ (complete response, $15.3 \%$; partial response, $30.5 \%$ ). The median DOR was 17.3 months, and the median PFS was 3.6 months. Grade 3 or 4 hematological toxicities included neutropenia (36\%), leukopenia (29\%), thrombocytopenia $(22 \%)$, and anemia (12\%). The combination of bendamustine and rituximab showed modest activity in patients with relapsed and refractory DLBCL and has an acceptable toxicity profile.

Keywords Diffuse large B cell lymphoma - Bendamustine · Alkylating agents $\cdot$ Rituximab $\cdot$ CD20 $\cdot$ Chemotherapy ·

Aggressive lymphoma

\section{Introduction}

Diffuse large B cell lymphoma (DLBCL) is the most common subtype of non-Hodgkin's lymphoma (NHL), accounting for approximately $30 \%$ of all lymphoma diagnoses. It is a heterogeneous disorder with subtypes distinguished by various clinical, pathologic, and molecular characteristics [1]. Since the 1970s, the combination of cyclophosphamide, vincristine, doxorubicin, and prednisone (CHOP) has been the standard therapy for DLBCL in the USA [2], producing long-term 5year survival in 30-35\% of patients with other more intensive drug combinations producing no additional benefit [3]. In 2002, the addition of the chimeric anti-CD20 monoclonal antibody rituximab to CHOP (R-CHOP) was shown to significantly improve both the complete response (CR) rate and overall survival (OS) of patients with DLBCL, with 5-year OS of $58 \%$ for R-CHOP versus $45 \%$ for CHOP alone [4]. The combination of R-CHOP has since become the most broadly accepted treatment regimen for DLBCL.

Patients who are refractory to induction therapy or relapse after achieving CR may be considered for salvage chemotherapy and, if their disease is chemosensitive, for high-dose 
chemotherapy and autologous stem cell transplant (ASCT). Regimens utilized in the salvage setting, which tend to be poorly tolerated and are frequently associated with treatmentrelated complications, include combinations of ifosfamide, carboplatin, and etoposide (ICE); dexamethasone, cytosine arabinoside, and cisplatin (DHAP); or etoposide, methylprednisolone, cytosine arabinoside, and cisplatin (ESHAP). Rituximab is typically included as part of the salvage treatment, despite the likelihood that most patients would have received rituximab in prior treatment settings [5-7]. Factors that can adversely affect the outcome after ASCT include duration of response less than 12 months, International Prognostic Index (IPI) greater than 1, and having received rituximab as part of initial treatment [8]. For those patients who are not candidates for ASCT due to advanced age, comorbidities, or chemorefractory disease, treatment options are limited, and prognosis is poor.

Bendamustine, a drug developed in the former East Germany, is an alkylating agent that appears to overcome cross-resistance to other alkylators and contains a benzimidazole ring, which may confer its unique properties [9]. In the USA, bendamustine is approved for the treatment of CLL and rituximab-refractory indolent NHL [10]. In Germany, the combination of bendamustine and rituximab (BR) has been directly compared with R-CHOP by Rummel and colleagues. The results of this trial indicate that the $\mathrm{BR}$ regimen produced a higher CR rate than R-CHOP (40.1 vs. $30.8 \%$ ), along with improved progression-free survival (69.5 vs. 31.2 months). This study was aimed at the indolent NHL as well as mantle cell lymphoma but did not include aggressive NHL such as DLBCL [11, 12].

Additional studies have also demonstrated activity for bendamustine in Hodgkin's lymphoma [13, 14], multiple myeloma, and various solid tumors [9]. However, prospective trials specifically investigating the efficacy of BR in patients with DLBCL were sparse, and for that reason, we undertook a phase II trial aimed at patients with DLBCL who are deemed to be inappropriate candidates for intensive salvage therapies.

Patients and methods

Patient selection

Patients with histologically confirmed CD20-positive DLBCL who relapsed or were refractory to at least one prior therapeutic regimen were eligible for this study. Measurable disease defined as at least one tumor of $>1.5 \mathrm{~cm}$ in the longest diameter was required. Eligible patients were required to have an Eastern Cooperative Oncology Group (ECOG) performance status of 0 to 2; adequate hematologic, renal, and hepatic functions; and life expectancy of at least 3 months. Autologous stem cell transplant-eligible patients were excluded, with the exception of those who refused ASCT. Patients with disease relapse after prior ASCT or allogeneic stem cell transplantation were allowed. Study patients were not candidates for high-dose therapy either because of advanced age, failure of salvage therapy, or comorbidities. Patients were naïve to bendamustine treatment.

Additional key exclusion criteria include known active infection with human immunodeficiency virus, hepatitis B virus, or hepatitis $C$ virus; history of another active malignancy within 1 year of study entry except for nonmelanoma skin cancer or carcinoma in situ of the cervix or breast; grade 3 or 4 active intercurrent bacterial infection requiring treatment or systemic fungal infection; and myocardial infarction within 6 months or New York Heart Association class III or IV heart failure, uncontrolled angina, ventricular arrhythmia, electrocardiographic evidence of acute ischemia, or significant conduction system abnormalities.

\section{Ethical oversight}

All patients provided written informed consent prior to any study-related procedures or assessments. The trial was conducted under review of an institutional review board for each site, in accordance with the 1964 Declaration of Helsinki and its later amendments, US Food and Drug Administration Good Clinical Practices, and local ethical and legal requirements. The study utilized a Data Monitoring Committee to adjudicate treatment safety and efficacy data of the two-stage design.

\section{Treatment administration}

Study patients received intravenous bendamustine and rituximab in 28-day cycle for up to 6 cycles. The dose of bendamustine was $90 \mathrm{mg} / \mathrm{m}^{2}$ administered on days 1 and 2 of each cycle for the first two patients on study. Following the US Food and Drug Administration (FDA) approval of the indication for bendamustine in relapsed, indolent non-Hodgkin's lymphoma, the dosage of bendamustine was amended to $120 \mathrm{mg} / \mathrm{m}^{2}$ administered on days 1 and 2 of each 21 -day cycle for all other patients on study. Rituximab was administered at a dose of $375 \mathrm{mg} / \mathrm{m}^{2}$ on day 1 of each cycle to all patients. Bendamustine was provided by Cephalon, Inc. (Malvern, PA). Rituximab was obtained through commercial sources. Prophylactic administration of colony-stimulating factor in cycle 1 was proscribed. Otherwise, the use of supportive and prophylactic concomitant medicines followed standard practice, at investigator's discretion.

Decisions for treatment delay and dose modification were based on treatment emergent adverse events as graded by the National Cancer Institute's Common Terminology Criteria for Adverse Events (version 3.0) and coded according to the Medical Dictionary for Regulatory Activities (MedRA; version 10.0) $[15,16]$. In the event of grade 4 hematologic toxicity 
or clinically significant non-hematologic toxicity of $\geq$ grade 2 on the day of intended bendamustine treatment, bendamustine administration was delayed until recovery of non-hematologic toxicity to $\leq$ grade 1 or improvement of blood counts to absolute neutrophil count of $>1 \times 10^{9} / \mathrm{L}$ and platelets of $>75 \times 10^{9} / \mathrm{L}$. Bendamustine could be delayed for up to 21 days and was dose modified in accordance with the grade and occurrence of the adverse event. At first occurrence of a grade 4 hematologic toxicity or clinically significant non-hematologic toxicity of $\geq$ grade 3 at any point during a cycle, bendamustine was dose reduced to $90 \mathrm{mg} / \mathrm{m}^{2}$ for all subsequent cycles. At second occurrence of a grade 4 hematologic toxicity or clinically significant non-hematologic toxicity of $\geq$ grade 3 at any point during a cycle, bendamustine was to be further dose reduced to $60 \mathrm{mg} / \mathrm{m}^{2}$ for all subsequent cycles. Study treatment was to be discontinued upon the third occurrence of a grade 4 hematologic toxicity or clinically significant non-hematologic toxicity of $\geq$ grade 3 at any point during a cycle. Treatment continued until completion of 6 cycles of therapy, disease progression, unacceptable toxicity, or patient withdrawal of consent.

\section{Response assessments and criteria}

After providing informed consent, study patients underwent baseline tests which included physical examination, vital signs, ECOG performance status, bone marrow aspirate and biopsy, positron emission tomography and computed tomography scan, revised IPI score documentation, electrocardiography, hematology and serum chemistry panels, hepatitis B screening, and pregnancy test. Response assessment was determined on study in accordance with the revised response criteria for malignant lymphoma [17]. Hematology and serum chemistry, adverse events reporting, and concomitant medication documentation were performed on days $1,2,8,15$, and 22 of cycles 1 to 6 . Physical examination and ECOG performance status assessment were performed on day 1 of each treatment cycle. Final safety assessment was performed 10 to 12 weeks following the last dose of study treatment. Restaging scans and disease response assessment were performed after every two cycles and at 3-month intervals during follow-up for up to 3 years.

\section{Objectives and statistical design}

The primary objective was to estimate the overall response rate (ORR). Secondary objectives included the duration of response (DOR), progression-free survival (PFS), OS, and treatment safety. The Simon two-stage design was followed, with a planned enrollment of at least 54 patients $\left(a=0.1, b=0.2, P_{0}\right.$ $=50 \%, P_{1}=70 \%$ ) [18]. During the first stage, treatment safety was evaluated by interim analysis $(n=5)$. In addition, if 8 or more of the first 15 patients $(\geq 53.3 \%)$ demonstrated objective response, then the second stage could be completed, to total sample size of at least 43 patients evaluable for ORR. The statistical design utilized an exact two-stage binomial test design, assuming $50 \%$ ORR as the null hypothesis and the alternative proportion (study ORR) of $70 \%$ ORR, with 0.05 one-sided significance and $80 \%$ power.

ORR was estimated as the proportion of patients who received any dose of bendamustine, defined for this singlearm study as the intent-to-treat (ITT) population, with the best overall response of CR or partial response (PR). The probability of response in each category was estimated as the proportion of patients in the ITT population attaining each response outcome. Clopper-Pearson two-sided $95 \%$ confidence intervals were calculated. Duration of response was measured as the time from the first documented response to the date of disease progression. Progression-free survival was measured as the time from the start of treatment to the date of disease progression or death as a result of any cause. Secondary efficacy endpoints were censored at 3 years after the start of treatment. The time to event data was estimated by KaplanMeier methods [19]. Adverse events were tabulated by patient incidence and grade. Percentages were calculated as the proportion of patients in the safety population experiencing an adverse event. The safety population included all patients who received at least one dose of study medication.

\section{Results}

\section{Patient characteristics}

Sixty-one patients were enrolled into the trial between December 2008 and January 2011, by 26 study centers in the Pharmatech Research Network. All patients were determined eligible for the study. Two patients withdrew prior to study treatment. Fifty-nine patients were evaluated in the ITT population. Patient characteristics are shown in Table 1. Study patients had a median age of 74, evenly distributed for gender, and $94 \%$ had ECOG 0 or 1 . Eighty-nine percent of patients had Ann Arbor stage III or IV disease, and $63 \%$ had a poor revised IPI risk score. Bone marrow involvement was positive in $11 \%$ of patients. Fifty-one percent of patients had received one prior line of therapy, $21 \%$ had received two, $13 \%$ had received three, and $15 \%$ had received more than three prior lines of therapy. Almost all patients had received prior rituximab (95\%). Five patients (8\%) had undergone prior ASCT.

Patient disposition is shown in Table 2. A total of 203 cycles of treatment were given to 59 patients on study, with a median of 4 cycles completed (range, 1-6 cycles). The distribution of patients by number of cycles completed is depicted. Seventeen patients $(29 \%)$ required reduction of bendamustine to the $90 \mathrm{mg} / \mathrm{m}^{2}$ dosage level, such that the mean dosage intensity of bendamustine was $233 \mathrm{mg} / \mathrm{m}^{2}$ per cycle or $97 \%$ of the 
Table 1 Patient demographics and clinical characteristics

\begin{tabular}{|c|c|c|}
\hline Characteristic & $n$ & Percent \\
\hline Total enrolled & 61 & 100 \\
\hline Safety population & 59 & 97 \\
\hline \multicolumn{3}{|l|}{ Age, years } \\
\hline Median (range) & $74(25-90)$ & \\
\hline$<60$ & 9 & 15 \\
\hline$\geq 60$ & 52 & 85 \\
\hline \multicolumn{3}{|l|}{ Gender } \\
\hline Male & 30 & 49 \\
\hline Female & 31 & 51 \\
\hline \multicolumn{3}{|l|}{ Ann Arbor stage } \\
\hline $\mathrm{I}-\mathrm{II}$ & 6 & 10 \\
\hline III-IV & 54 & 89 \\
\hline Unknown & 1 & 1 \\
\hline \multicolumn{3}{|l|}{ ECOG PS } \\
\hline 0 & 26 & 43 \\
\hline 1 & 31 & 51 \\
\hline 2 & 4 & 6 \\
\hline \multicolumn{3}{|c|}{ Bone marrow involvement } \\
\hline Positive & 7 & 11 \\
\hline Negative & 51 & 84 \\
\hline Unknown & 3 & 5 \\
\hline \multicolumn{3}{|c|}{ Prior lines of therapy } \\
\hline 1 & 31 & 51 \\
\hline 2 & 13 & 21 \\
\hline 3 & 8 & 13 \\
\hline$>3$ & 8 & 15 \\
\hline Median (range) & $1(1-9)$ & \\
\hline \multicolumn{3}{|c|}{ Prior rituximab therapy } \\
\hline Yes & 58 & 95 \\
\hline No & 3 & 5 \\
\hline \multicolumn{3}{|c|}{ Prior autologous stem cell transplant } \\
\hline Yes & 5 & 8 \\
\hline No & 56 & 92 \\
\hline \multicolumn{3}{|l|}{ RIPI risk group } \\
\hline Very good & 0 & 0 \\
\hline Good & 22 & 36 \\
\hline Poor & 38 & 63 \\
\hline Unknown & 1 & 1 \\
\hline
\end{tabular}

planned dosage intensity of $240 \mathrm{mg} / \mathrm{m}^{2}$ per cycle. No patient required a second bendamustine dose reduction. The dosage of rituximab was not reduced, and $100 \%$ of the planned dosage of $375 \mathrm{mg} / \mathrm{m}^{2}$ rituximab per cycle was administered. All patients have either completed or discontinued treatment. Reasons for not completing the full 6 cycles of treatment included 26 patients ( $44 \%$ ) with disease progression, 4 patients $(8 \%)$ withdrew consent, 4 patients $(8 \%)$ with intercurrent illness, and 4 patients $(8 \%)$ died on study.
Table 2 Patient disposition

\begin{tabular}{|c|c|c|}
\hline Endpoint & $n$ & Percent \\
\hline Patients enrolled & 61 & 100 \\
\hline Patients treated & 59 & 97 \\
\hline Number of cycles completed & 203 & \\
\hline Mean (range) & $3(1-$ & \\
\hline \multicolumn{3}{|l|}{ Patients completing } \\
\hline 1 & 59 & 29 \\
\hline 2 & 50 & 25 \\
\hline 3 & 36 & 18 \\
\hline 4 & 29 & 14 \\
\hline 5 & 17 & 8 \\
\hline 6 & 12 & 6 \\
\hline Bendamustine dosage per cycle & \multicolumn{2}{|c|}{$180 \mathrm{mg} / \mathrm{m}^{2}\left(90 \mathrm{mg} / \mathrm{m}^{2} \times 2\right)$} \\
\hline Patients & 2 & 3 \\
\hline Mean administered (range) & \multicolumn{2}{|c|}{$180 \mathrm{mg} / \mathrm{m}^{2}(180)$} \\
\hline Bendamustine dosage per cycle & \multicolumn{2}{|c|}{$240 \mathrm{mg} / \mathrm{m}^{2}\left(120 \mathrm{mg} / \mathrm{m}^{2} \times 2\right)$} \\
\hline Patients & 57 & 93 \\
\hline Mean administered (range) & \multicolumn{2}{|c|}{$234 \mathrm{mg} / \mathrm{m}^{2}(120-240)$} \\
\hline Rituximab dosage & \multicolumn{2}{|c|}{$375 \mathrm{mg} / \mathrm{m}^{2}$} \\
\hline Patients & 59 & 97 \\
\hline Mean administered (range) & \multicolumn{2}{|c|}{$375 \mathrm{mg} / \mathrm{m}^{2}(375)$} \\
\hline \multicolumn{3}{|c|}{$\begin{array}{l}\text { Reasons for treatment discontinuation } \\
\text { in patients receiving }<6 \text { cycles }\end{array}$} \\
\hline Progressive disease & 26 & 53 \\
\hline Investigator decision & 6 & 13 \\
\hline Patient expired & 4 & 8 \\
\hline Disease related & 2 & \\
\hline Disseminated herpes zoster & 1 & \\
\hline Unknown cause & 1 & \\
\hline Intercurrent illness & 4 & 8 \\
\hline Patient withdrew consent & 4 & 8 \\
\hline Patient is lost to follow-up & 1 & 2 \\
\hline Protocol noncompliance & 1 & 2 \\
\hline Non-protocol therapy given & 1 & 2 \\
\hline Irradiation of target lesion & 1 & 2 \\
\hline Unknown & 1 & 2 \\
\hline
\end{tabular}

Treatment safety

In the first stage of the study, five patients were assessed by a data safety committee following completion of at least 1 cycle of therapy. After the first two patients, the bendamustine dose was amended to $120 \mathrm{mg} / \mathrm{m}^{2}$, concurrent with the US FDA approval for indolent, relapsed non-Hodgkin's lymphoma. This dose was determined by the safety committee to have a high probability of acceptable tolerability without a separate dose escalation study. The most common reasons for bendamustine dose reduction were neutropenia and thrombocytopenia. Other adverse events included anorexia, 
dehydration, fatigue, hemoglobin, leukopenia, mucositis, shingles, and weight loss. Adverse event data are presented, according to hematologic (Table 3) and non-hematologic (Table 4) events. Events which occurred in $\geq 10 \%$ of patients are reported for all grades combined, grade 3 events, and grade 4 events. The most common hematologic adverse event reported in the study was neutropenia, thrombocytopenia, and anemia, each occurring in $44 \%$ of patients. Grade 4 adverse events included febrile neutropenia in four patients (7\%), thrombocytopenia in three patients (5\%), and leukopenia in one patient (2\%). Grade 3 adverse events included neutropenia in 17 patients (29\%), thrombocytopenia in 10 patients (17\%), leukopenia in 13 patient $(22 \%)$, and anemia in 7 patients (12\%). Four deaths occurred on during the study, including two patients who died from disease-related complications, one patient from disseminated herpes zoster, and one patient of unknown cause.

\section{Efficacy}

In stage 1 of the study, 9 of the first 15 patients treated $(60 \%)$ achieved an objective response; therefore, the study was allowed to complete the enrollment. The final study ORR was $45.8 \%$ (27/59 patients; $95 \%$ confidence interval (CI), 41-70\%), including $15.3 \% \mathrm{CR}$ and $30.5 \% \mathrm{PR}$, shown in Table 5. Stable disease was reported in $18.6 \%$ percent of patients, while $16.9 \%$ progressed on treatment or withdrew prior to evaluation. While the observed ORR is clinically significant, it did not meet the statistical endpoint of $70 \%$ response rate for $\mathrm{BR}$ in this treatment population.

Kaplan-Meier regression of event-free outcomes and DOR are depicted in Fig. 1. Median DOR among 27 patients with an objective response was 17.3 months (Fig. 1a; $95 \%$ CI, 4.2 months; upper limit not reached). Response was maintained for 15 patients at the data censor date. Median PFS was 3.6 months (Fig. 1b; 95 \% CI, 2.7-7.2 months). Eighteen patients remained progression free at the data censor date. Patient overall survival was evaluated for the 3-year period beginning with the initiation of study treatment. Due to a high

Table 3 Hematologic adverse events occurring in DLBCL patients receiving bendamustine and rituximab

\begin{tabular}{|c|c|c|c|c|c|c|}
\hline \multirow[t]{2}{*}{ Event } & \multicolumn{2}{|c|}{ All grades } & \multicolumn{2}{|c|}{ Grade 3} & \multicolumn{2}{|c|}{ Grade 4} \\
\hline & $n$ & Percent & $N$ & Percent & $n$ & Percent \\
\hline Neutropenia & 26 & 44 & 17 & 29 & 4 & 7 \\
\hline Leukopenia & 20 & 34 & 13 & 22 & 1 & 2 \\
\hline Thrombocytopenia & 26 & 44 & 10 & 17 & 3 & 5 \\
\hline Anemia & 26 & 44 & 7 & 12 & 0 & 0 \\
\hline
\end{tabular}

Severity was determined from post-baseline laboratory results using the National Cancer Institute Common Terminology Criteria for Adverse Events, version 3.0, and MedRA
Table 4 Non-hematologic adverse events occurring in DLBCL patients receiving bendamustine and rituximab. Censored at $10 \%$

\begin{tabular}{|c|c|c|c|c|c|c|}
\hline \multirow[t]{2}{*}{ Event } & \multicolumn{2}{|c|}{ All grades } & \multicolumn{2}{|c|}{ Grade 3} & \multicolumn{2}{|c|}{ Grade 4} \\
\hline & $n$ & Percent & $N$ & Percent & $n$ & Percent \\
\hline Nausea & 24 & 41 & 1 & 2 & 1 & 2 \\
\hline Fatigue & 21 & 36 & 2 & 3 & 0 & 0 \\
\hline Diarrhea & 18 & 30 & 2 & 3 & 0 & 0 \\
\hline Fever & 17 & 29 & 1 & 2 & 1 & 2 \\
\hline Anorexia & 14 & 24 & 3 & 5 & 0 & 0 \\
\hline Constipation & 13 & 22 & 0 & 0 & 0 & 0 \\
\hline Cough & 11 & 19 & 1 & 2 & 0 & 0 \\
\hline Weight loss & 11 & 19 & 1 & 2 & 0 & 0 \\
\hline Vomiting & 10 & 17 & 1 & 2 & 1 & 2 \\
\hline Dizziness & 9 & 15 & 2 & 3 & 0 & 0 \\
\hline Abdominal pain & 8 & 14 & 3 & 5 & 0 & 0 \\
\hline Limb edema & 8 & 14 & 1 & 2 & 0 & 0 \\
\hline Pain & 8 & 14 & 0 & 0 & 0 & 0 \\
\hline Back pain & 7 & 12 & 1 & 2 & 0 & 0 \\
\hline Chills & 6 & 10 & 0 & 0 & 0 & 0 \\
\hline Dehydration & 6 & 10 & 2 & 3 & 0 & 0 \\
\hline Dyspnea & 6 & 10 & 3 & 5 & 0 & 0 \\
\hline Headache & 6 & 10 & 1 & 2 & 0 & 0 \\
\hline Hypotension & 6 & 10 & 0 & 0 & 1 & 2 \\
\hline
\end{tabular}

Severity was determined from post-baseline laboratory results using the National Cancer Institute Common Terminology Criteria for Adverse Events, version 3.0, and MedRA

number of censored data, as patients withdrew from study follow-up, the median OS was not reached (data not shown).

\section{Discussion}

The treatment of DLBCL changed dramatically with the landmark GELA trial which demonstrated dramatic response and survival advantages with the addition of rituximab to the established standard, CHOP. Nevertheless, a significant proportion of patients remains either refractory to or relapses after R-CHOP therapy. Depending on the stage of the disease, the clinical IPI scores, and biologic characteristics, up to $40 \%$ of

Table 5 Treatment response

\begin{tabular}{lrl}
\hline Response category & $n$ & Percent $(n=59)$ \\
\hline ORR & 27 & 45.8 \\
CR & 9 & 15.3 \\
PR & 18 & 30.5 \\
SD & 11 & 18.6 \\
PD & 10 & 16.9 \\
\hline
\end{tabular}



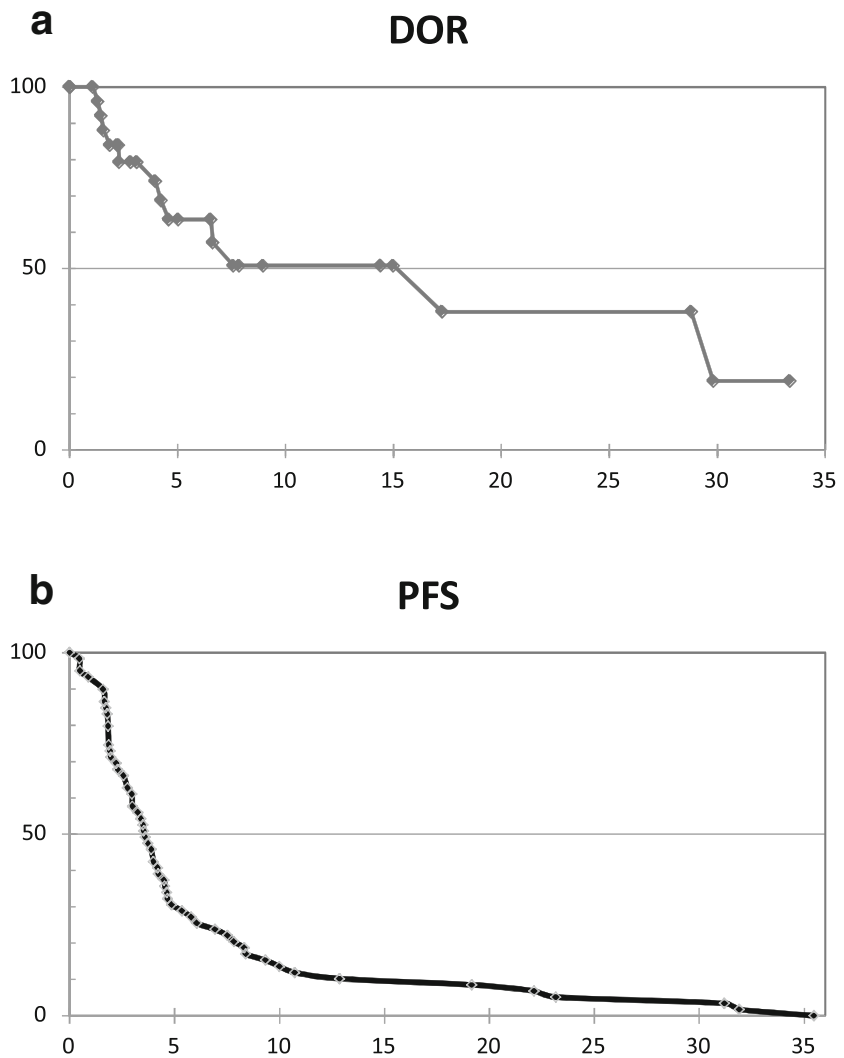

Fig. 1 Kaplan-Meier time to event curves. Vertical axis represents the percentage of patients. Horizontal axis depicts time in months. a Duration of response (median=17.3 months). b Progression-free survival (median=3.6 months)

patients may not be cured. Certain subtypes of DLBCL, such as the so-called double-hit lymphomas, have a particularly ominous prognosis [20]. The standard of care for chemosensitive, medically fit relapsed or refractory patients has been ASCT. Paradoxically, in the rituximab era, the outcome following ASCT has worsened. Gisselbrecht has defined adverse prognostic factors for survival following ASCT such as prior rituximab (which includes virtually all patients), short duration of response ( $<12$ months), and high IPI score at relapse ( $>1)$ [8]. Taken together, the overall cure rate following ASCT is probably no greater than $20 \%[8,21]$. New agents which may have impact on the management of DLBCL include the immunoconjugates [22], B cell receptor [23] kinase inhibitors such as agents inhibiting PI3 kinase [24], Bruton's tyrosine kinase [25], splenic tyrosine kinase [26], and immunomodulators such as lenalidomide [27].

In our study, we examined a group of patients who had a median age of 74 years and who were determined by their physicians not to be good candidates for ASCT or aggressive salvage regimens. Bendamustine, a novel alkylating agent that demonstrates a lack of cross-resistance with standard alkylators, has shown a significant activity in a variety of lymphoproliferative diseases, including chronic lymphocytic leukemia and indolent lymphomas. More recently, activity has been demonstrated in multiple myeloma and Hodgkin's lymphoma. Aggressive lymphomas such as DLBCL have been less extensively studied. In our trial, the combination of bendamustine and rituximab produced an ORR of $45.8 \%$ with complete responses in $15.3 \%$ of patients. The median duration of response was 17.3 months with an intention-totreat progression-free survival of 3.6 months. While these results are inferior to the results anticipated with more aggressive salvage regimens such as R-ESHAP and R-ICE, the intent-to-treat population of our study was patients who were poor candidates by age and medical criteria for such aggressive therapy. A review of previous experiences in aggressive lymphomas using bendamustine includes a small number of manuscripts. Weidmann et al. reported an ORR of $44 \%$ utilizing single-agent bendamustine in a study of 21 patients [28]. Rigacci et al. published a small study experience including bendamustine with or without rituximab in a variety of NHL subtypes [29]. In 34 patients with DLBCL, the ORR was $33 \%$ with $12 \% \mathrm{CR}$, which is similar to our experience. In a small study reported as a letter to the editor, Walter et al. noted a $57 \%$ ORR (CR $29 \%$ ) in eight relapsed patients, while no patient with refractory disease demonstrated a response [30]. Horn et al. treated a total of 20 patients with so-called aggressive lymphoma, $75 \%$ of whom had DLBCL [31]. The ORR in that trial was $55 \%$. In that study of frail and elderly patients, both previously untreated and relapsed patients were included. Finally, a very recent phase II trial in DLBCL reported a higher response rate of $63 \%$ (CR $37 \%$ ), using $120 \mathrm{mg} / \mathrm{m}^{2}$ bendamustine with rituximab, which is the most favorable experience yet with this combination [32]. Other alternatives to be considered in the relapsed/refractory population of DLBCL include the gemcitabine-oxaliplatin combination [33]. The results of this combination may be somewhat better than reported for $\mathrm{BR}$, but it is premature to compare regimens in the phase II setting.

Despite the fact that most of our patients received a dose of $120 \mathrm{mg} / \mathrm{m}^{2}$ bendamustine, higher than the usual dose of $90 \mathrm{mg} /$ $\mathrm{m}^{2}$ used in rituximab combinations, the regimen was reasonably well tolerated, although a dose reduction was employed in a third of our patients. Only $7 \%$ of patients developed febrile neutropenia. In summary, the combination of bendamustine and rituximab showed modest activity in DLBCL; in an adverse population of patients not felt suitable for transplantation, only 5 of the 61 patients enrolled had undergone prior ASCT. The role of bendamustine in treating both newly diagnosed and progressive DLBCL bears further study. Ongoing trials are evaluating bendamustine in combination with rituximab, ofatumumab, or rituximab with lenalidomide in untreated patients who are not candidates for R-CHOP. A recent experience including treatment-naïve patients over age 80 showed encouraging results [31]. Further combinations utilizing bendamustine in the salvage setting with additional 
drugs, as well as pre-transplant conditioning regimens, are also being evaluated in clinical trials.

Acknowledgments Sincere thanks to all of the patients and physicians who participated in this study. This research was wholly supported by an investigator-sponsored trial grant from Cephalon, Inc. Cephalon, Inc. provided bendamustine for patients on study.

Conflict of interest The authors declare that they have no conflict of interest.

Open Access This article is distributed under the terms of the Creative Commons Attribution License which permits any use, distribution, and reproduction in any medium, provided the original author(s) and the source are credited.

\section{References}

1. Jaffe ES, Harris NL, Stein H et al (2008) Classification of lymphoid neoplasms: the microscope as a tool for disease discovery. Blood 112:4384-4399

2. Jones SE, Grozea PN, Miller TP et al (1985) Chemotherapy with cyclophosphamide, doxorubicin, vincristine, and prednisone alone or with levamisole or with levamisole plus BCG for malignant lymphoma: a Southwest Oncology Group study. J Clin Oncol 3:1318-1324

3. Fisher RI, Gaynor ER, Dahlberg S et al (1993) Comparison of a standard regimen (CHOP) with three intensive chemotherapy regimens for advanced non-Hodgkin's lymphoma. N Engl J Med 328:1002-1006

4. Feugier P, Van Hoof A, Sebban C et al (2005) Long-term results of the R-CHOP study in the treatment of elderly patients with diffuse large B-cell lymphoma: a study by the Groupe d'Etude des Lymphomes de l'Adulte. J Clin Oncol 23:4117-4126

5. Mey UJ, Orlopp KS, Flieger D et al (2006) Dexamethasone, highdose cytarabine, and cisplatin in combination with rituximab as salvage treatment for patients with relapsed or refractory aggressive non-Hodgkin's lymphoma. Cancer Invest 24:593-600

6. Harting R, Venugopal P, Gregory SA et al (2007) Efficacy and safety of rituximab combined with ESHAP chemotherapy for the treatment of relapsed/refractory aggressive B-cell non-Hodgkin lymphoma. Clin Lymphoma Myeloma 7:406-412

7. López A, Gutierrez A, Palacios A et al (2008) GEMOX-R regimen is a highly effective salvage regimen in patients with refractory/ relapsing diffuse large-cell lymphoma: a phase II study. Eur J Haematol 80:127-132

8. Gisselbrecht C, Glass B, Mounier N et al (2010) Salvage regimens with autologous transplantation for relapsed large B-cell lymphoma in the rituximab era. J Clin Oncol 28:4184-4190

9. Cheson BD, Rummel MJ (2009) Bendamustine: rebirth of an old drug. J Clin Oncol 27:1492-1501

10. Ujjani C, Cheson B (2011) Efficacy of bendamustine in rituximabrefractory indolent B-cell non-Hodgkin lymphoma: review of a pivotal trial. Future Oncol 1:9-14

11. Rummel MJ, Niederle N, Maschmeyer G et al (2009) Bendamustine plus rituximab is superior in respect of progression free survival and $\mathrm{CR}$ rate when compared to $\mathrm{CHOP}$ plus rituximab as first-line treatment of patients with advanced follicular, indolent, mantle cell lymphomas: final results of a randomized phase III study of the StiL (Study Group Indolent Lymphomas, Germany). Blood 114, Abstract 405

12. Rummel MJ, Niederle N, Maschmeyer G et al (2012) Bendamustine plus rituximab (B-R) versus $\mathrm{CHOP}$ plus rituximab (CHOP-R) as first-line treatment in patients with indolent and mantle cell lymphomas (MCL): updated results from the StiL NHL1 study. J Clin Oncol 30, Abstract 3

13. Moskowitz AJ, Hamlin PA, Gerecitano J et al (2009) Bendamustine is highly active in heavily pre-treated relapsed and refractory Hodgkin lymphoma and serves as a bridge to allogeneic stem cell transplant. Blood 114, Abstract 720

14. D'Elia GM, De Angelis F, Breccia M et al (2010) Efficacy of bendamustine as salvage treatment in an heavily pre-treated Hodgkin lymphoma. Leuk Res 34(11):300-301

15. DCTD, NCI, NIH, DHHS (2006) Cancer Therapy Evaluation Program: common terminology criteria for adverse events, version 3.0. http://ctep.cancer.gov/protocolDevelopment/electronic_applications/ docs/ctcaev3.pdf. Accessed 6 June 2012

16. MedDRA (2008) Medical dictionary for regulatory activities. Version 10.0. Northrop Grumman Corporation, Los Angeles. www. meddramsso.com. Accessed 6 June 2012. Available by subscription

17. Cheson BD, Pfistner B, Juweid ME, Gascoyne RD, Specht L (2007) Revised response criteria for malignant lymphoma. J Clin Oncol 26:579-86

18. Simon R (1989) Optimal two-stage designs for phase II clinical trials. Control Clin Trials 10:1-10

19. Kaplan E, Meier P (1958) Nonparametric estimation from incomplete observations. J Am Stat Assoc 53:457-481

20. Aukema SM, Siebert R, Schuuring E et al (2011) Double-hit B-cell lymphomas. Blood 117:2319-2331

21. Friedberg JW (2008) Diffuse large B-cell lymphoma. Hematol Oncol Clin North Am 22(5):941-952

22. Lee PP, Rosen PJ (2011) Radioimmunotherapy for CD20-positive Bcell non-Hodgkin's lymphoma. Commun Oncol 8:24-31

23. Cheng S, Coffey G, Zhang XH et al (2011) SYK inhibition and response prediction in diffuse large B-cell lymphoma. Blood 118:6342-6352

24. Lannutti BJ, Meadows SA, Herman SE et al (2011) CAL-101, a p110delta selective phosphatidylinositol-3-kinase inhibitor for the treatment of B-cell malignancies, inhibits PI3K signaling ad cellular viability. Blood 117:591-594

25. Habermann TM (2012) New developments in the management of diffuse large B-cell lymphoma. Hematol Suppl 1:S93-7

26. Choi MY, Kipps TJ (2012) Inhibitors of B-cell receptor signaling for patients with B-cell malignancies. Cancer J 18(5):404-410

27. Hernandez-Ilizaliturri FJ, Deeb G, Zinzani PL et al (2011) Higher response to lenalidomide in relapsed/refractory diffuse large B-cell lymphoma in nongerminal center B-cell-like than in germinal center B-cell-like phenotype. Cancer 117:5058-5066

28. Weidmann E, Kim SZ, Rost A et al (2002) Bendamustine is effective in relapsed or refractory aggressive non-Hodgkin's lymphoma. Ann Oncol 13(8):1285-1289

29. Rigacci L, Puccini B, Cortelazzo S et al (2012) Bendamustine with or without rituximab for the treatment of heavily pretreated nonHodgkin's lymphoma patients. Ann Hematol 91:1013-1022

30. Walter E, Schmitt T, Dietrich S et al (2012) Rituximab and bendamustine in patients with $\mathrm{CD} 20^{+}$diffuse large B-cell lymphoma not eligible for cyclophosphamide, doxorubicin, vincristine, and prednisone-like chemotherapy. Leuk Lymphoma 53(11):2290-2292

31. Horn J, Kleber M, Hieke S et al (2012) Treatment option of bendamustine in combination with rituximab in elderly and frail patients with aggressive B-non-Hodgkin lymphoma: rational, efficacy, and tolerance. Ann Hematol 91(10):1579-1586

32. Ohmachi K, Niitsu N, Uchida T et al (2013) Multicenter phase II study of bendamustine plus rituximab in patients with relapsed or refractory diffuse large B-cell lymphoma. J Clin Oncol 31(17):2103-2109

33. Corazzeli G, Capobianco G, Arcamone M et al (2009) Long-term results of gemcitabine plus oxaliplatin with and without rituximab as salvage treatment for transplant-ineligible patients with refractory/relapsing Bcell lymphoma. Cancer Chemother Pharmacol 64(5):907-916 